\title{
STochastic Optical Reconstruction Microscopy (STORM) reveals the nanoscale organization of pathological aggregates in human brain
}

Philippe Codron ( $\nabla$ codron.ph@outlook.com )

Centre Hospitalier Universitaire d'Angers https://orcid.org/0000-0003-1745-8299

Franck Letournel

Centre Hospitalier Universitaire d'Angers

\section{Serge Marty}

Institut du cerveau et de la moelle epiniere

Alexia Bodin

Biologie neurovasculaire et mitochondriale integree

\section{Laurence Renaud}

Centre de recherche de l'Institut universitaire en sante mentale de Quebec

\section{Mathilde Duchesne}

Centre Hospitalier Universitaire de Limoges

\section{Christophe Verny}

Centre Hospitalier Universitaire d'Angers

\section{Guy Lenaers}

Biologie neurovasculaire et mitochondriale integree

\section{Charles Duyckaerts}

Institut du cerveau et de la moelle epiniere

Jean-Pierre Julien

Centre de recherche de l'Institut universitaire en sante mentale de Quebec

\section{Julien Cassereau}

Centre Hospitalier Universitaire d'Angers

\section{Arnaud Chevrollier}

Biologie neurovasculaire et mitochondriale integree

\section{Methodology}

Keywords: STORM, Tau, Amyloid- $\beta$, $\alpha$-synuclein, TDP-43, Neuropathology

Posted Date: April 22nd, 2020

DOI: https://doi.org/10.21203/rs.3.rs-22385/v1 
License: (c) (i) This work is licensed under a Creative Commons Attribution 4.0 International License. Read Full License

Version of Record: A version of this preprint was published at Neuropathology and Applied Neurobiology on August 12th, 2020. See the published version at https://doi.org/10.1111/nan.12646. 


\section{Abstract \\ Background}

Histological analysis of brain tissue samples provide valuable information about the pathological processes leading to common neurodegenerative disorders such as Alzheimer's or Parkinson's diseases. In particular, high resolution and specific analysis of intra-neuronal lesions is crucial to understand the pathogenesis and progression of these diseases. In this context, the development of novel imaging approaches is a current challenge in neuroscience.

\section{Methods}

To this end, we used a recent super-resolutive imaging technique called STochastic Optical Reconstruction Microscopy (STORM) to analyze human brain sections. We combined STORM cell imaging protocols with neuropathological techniques and imaged cryopreserved brain samples from control subjects and patients with neurodegenerative diseases.

\section{Results}

This approach allowed us to perform 2D-, 3D- and two-color-STORM in central nervous system tissue sections, and to characterize with a nanometer-scale precision the architecture of physiological and pathological structures in neocortex, white matter and brainstem samples. STORM proved to be particularly effective to visualize the organization of dense protein inclusions, and allowed us to image with unprecedented details $A \beta$, Tau, a-synuclein and TDP-43 pathological aggregates within the central nervous system of patients with neurodegenerative disorders.

\section{Conclusions}

STORM imaging of human brain samples opens further gates to a more comprehensive understanding of the underlying mechanisms responsible for common neurological diseases. The convenience of this technique should open a straightforward extension of its application for super-resolution imaging of the human brain, with promising avenues to current challenges in neuroscience.

\section{Background}

Advances in neuroscience are closely linked to the progresses made in cell imaging. The invention of the microscope in the 17th century first allowed the characterization of the human brain at the cellular level and the emergence of clinical neurology, neurosurgery and psychiatry in the 18th and 19th centuries. Likewise, the understanding and classification of neurodegenerative disorders benefited from the 
development of immunostaining techniques and multichannel fluorescence imaging in the second part of the 20th century.

However, despite these advances, the diffraction barrier $(\sim 250 \mathrm{~nm})$ remains a resolution limit for conventional light microscopes, hindering the precise characterization of subcellular structures.

Recently, super-resolution microscopy techniques overcame this limit by using different approaches [1]. One of them, called STochastic Optical Reconstruction Microscopy (STORM), is based on single molecule localization by stochastic excitation of photo-switchable fluorescent molecules [2]. By improving the resolution towards the nanometer scale ( 20-50 nm), STORM allows the visualization of nanoscopic structures while retaining the advantages of optical microscopy such as sample preparation, molecular identification through immunostaining, and multicolor fluorescence imaging [3]. In the field of neuroscience, this new technique led to the characterization of the periodic structure of axonal cytoskeleton in neurons [4] and the spatial organization of synaptic proteins [5]. However, to date, STORM has mainly been used to image cultured cells and rodent brain samples.

In this study, we combined cell imaging protocols with neuropathology techniques to perform 2D-, 3Dand two-color STORM on human brain samples from control subjects and patients with common agerelated neurodegenerative diseases.

\section{Results}

\section{Super-resolution imaging of human brain samples with STORM}

To assess STORM imaging on human brain tissue, we first aimed to resolve well-defined histological structures such as neocortical axon tracts. Frozen samples of prefrontal cortex from control subjects were cut by standard cryostat methods (Fig. S1) and immunostained with an anti-neurofilament primary antibody and a secondary antibody conjugated with the photo-switchable fluorophore Alexa Fluor (AF) 647. Coverslips with immunostained brain sections were bathed in a switching buffer (Fig. S1) and placed on the stage of an inverted motorized microscope equipped with a high-power laser module, a total internal reflection fluorescence (TIRF) system, and an electron multiplying charge coupled device (EMCCD) single-photon sensitive camera (Fig. 1A, Fig. S2).

STORM is based on separating stochastically the emission of photo-switchable fluorescent molecules in time, so that each individual emitter can be distinguished from the others that reside within the same diffraction-limited volume. For each acquisition, thousands of frames were recorded, and each frame was computationally processed to detect activated fluorescent molecules, determine their center position, and report it as a single pixel on a corresponding reconstruction picture. The final super-resolved STORM image was obtained by merging all the reconstruction pictures in a single overlap image (Fig. 1B, Video 
S1). The total acquisition and reconstruction time for one super-resolved image lasted from 5 to 10 minutes, depending on the number of acquired frames.

With the aim of assessing the final resolution of the technique, we imaged axon processes with conventional wide field fluorescence microscopy, STORM, and transmission electron microscopy (TEM) in prefrontal cortex samples of age-matched control subjects. Although neurofilament fibrils $(\sim 10 \mathrm{~nm})$ could not be defined as well as when using TEM, the caliber of axons imaged with STORM was lower than when using conventional wide field fluorescence microscopy (Fig. S3). For the same prefrontal axons, the apparent diameters and areas of longitudinally- and transversally-sectioned axons were more than $50 \%$ smaller when measured with STORM compared to wide field fluorescence microscopy $(p<$ 0.001 for the two parameters) (Fig. 1C-E, Tables S1 and S2). In contrast, there was no significant difference when comparing apparent diameters $(p=0.441)$ and areas $(p=0.596)$ measured with STORM and TEM (Fig. 1D,E, Tables S1 and S2). STORM is thus a reliable and resolutive technique allowing to image and analyze histological brain structures at the nanoscale level.

\section{D-STORM and two-color STORM imaging of human brain samples}

Three-dimensional (3D) and multichannel imaging provide fundamental insights into the architectural organization of nanoscale structures. To perform 3D-STORM in human brain sections we used an astigmatism-based method [6]. After setting a cylindrical lens in the detection path of the microscope, the $z$ coordinate of each fluorescent molecule was determined from the ellipticity of its image. Prefrontal cortex samples of control subjects were immuno-stained for neurofilaments (NF), and after reconstruction, axons were visualized in 3D with high resolution and an imaging depth of $\sim 0.5 \mu \mathrm{m}$ (Fig. 2A, Video S2). The cylindrical shape of axon tracts was discernible and both longitudinal and transversal sections of the 3D modelled axons were similar in size to those obtained with 2D-STORM.

Then, to assess two-color STORM imaging on cortex samples, we immuno-stained human brain sections with two primary antibodies detecting nearby structures, the pre- and post-synaptic Bassoon and Homer 1 proteins, and with secondary antibodies conjugated with the photoswitchable fluorophores AF 647 and AF 532 (Fig. 2B, Fig. S4). In conventional fluorescence microscopy, Bassoon and Homer 1 signals appeared diffuse and overlapping, and the synaptic clefts could not be precisely defined. In contrast, twocolor STORM accurately distinguished between pre- and post-synaptic protein clusters separated by the synaptic cleft, and defined the size, orientation and organization of the synapses. Interestingly, multichannel imaging combining conventional wide field fluorescence microscopy and STORM was also achievable, allowing super-resolution imaging of brain structures using both photoswitchable and nonphotoswitchable fluorophores. Although less specific than two-color STORM, this technique provides valuable information about the layout of adjacent structures, as for example the myelin sheath around axonal tracts (Fig. 2C, Fig. S4).

Together, these results demonstrate the possibility to perform 3D and two-color STORM imaging with a nanoscale resolution on human brain samples, providing valuable information to current topics in 
neuroscience, such as axonal organization, myelination and synaptic plasticity in the human brain.

Understanding the pathophysiology of neurodegenerative disorders to identify novel therapeutic prospects is another major challenge in neuroscience. Since most of these diseases are defined by specific intra- or extra-cellular protein aggregates in distinctive anatomical brain regions, novel insights must be disclosed on the precise characterization of the corresponding lesions. To this end, we performed STORM imaging of Amyloid- $\beta$ (A $\beta)$, Tau, $a$-synuclein and TDP-43 aggregates in samples from patients affected with neurodegenerative disorders.

\section{STORM imaging of Amyloid- $\beta$ and Tau proteinopathy}

Alzheimer's disease (AD) is the leading cause of dementia. The two main hallmarks of the disease are extracellular deposits of $A \beta$ peptides, some of which constituting the core of senile plaques, and intraneuronal aggregates of hyperphosphorylated tau protein (p.Tau) called neurofibrillary tangles (NFT) [7]. Since aggregates can measure up to $100 \mu \mathrm{m}$, keeping an overall view of the whole lesions is critical to study $A \beta$ and Tau pathology in the brain, while high resolution imaging is mandatory to characterize the nanoscale organization of the misfolded proteins.

Towards this goal, we imaged entire senile plaques and degenerating neurons with STORM. Tissue samples from the prefrontal, parietal and temporal cortex of $A D$ patients were immunostained for $A \beta$ and p.Tau (phospho Ser202, Thr205). Auto-fluorescence quenchers were used to reduce the signal of lipofuscin, an autofluorescent pigment present in senescent neurons [8]. Nevertheless, lipofuscin signal was specifically detectable in neuron but did not preclude STORM acquisitions.

STORM images of $\sim 30 \mu \mathrm{m}$ diameter senile plaques and $\sim 15 \mu \mathrm{m}$ degenerating neurons with NFTs were acquired. While the $A \beta$ fibrils and the paired helical filaments of Tau could not be identified as with TEM, STORM images provided highly resolved details of the nanoscale distribution and size of $A \beta$ and $p$.Tau aggregates (Fig. 3, Fig. S5). Aggregated $A \beta$ branches were reticulated and cross-linked in the extracellular matrix, and their widths ranged from 60 to $240 \mathrm{~nm}(140.8 \pm 39.6 \mathrm{~nm}$, mean \pm SD) (Fig. S6). Intraneuronal p.Tau NFTs appeared denser, with a honeycombed structure in the soma and a filamentous organization in the axon. The presence of unstained spots within the aggregates suggested the inclusion of other components, such as proteins or organelles. These results emphasize that STORM can be used to image $A \beta$ and p.Tau aggregates in brain samples from $A D$ patients with high resolution.

\section{STORM imaging of Lewy Pathology}

Parkinson's disease (PD) and dementia with Lewy bodies (DLB) are two neurodegenerative diseases characterized by the presence of intra-neuronal phosphorylated a-synuclein (p.a-syn) immuno-reactive inclusions, called Lewy bodies (LB) [9]. The structure of LBs varies according to their localization within the central nervous system. Two main LB types can be observed by immunohistochemistry: typical Lewy bodies (TLB) with a pale core surrounded by a dense halo mainly found in the brainstem, and smaller cortical Lewy bodies (CLB), lacking the central core and mainly detected in the neocortex. Accumulation 
of p.a-syn in dystrophic axons called Lewy neurites (LN) can also be observed. To date, the structure of LBs remains unclear, as the resolution of conventional fluorescence microscopy is too low to characterize their internal architecture and TEM does not provide sufficient information about their protein content and organization.

To characterize LB organization at the nanoscale level with a molecular staining approach, we performed STORM imaging on brain samples from PD and DLB patients. Substancia nigra and prefrontal cortex sections were immuno-stained with an anti-p.a-syn (phospho Ser129) antibody and images of TLBs, CLBs and LNs were acquired. The ring shaped appearance of TLBs were observed both with conventional and super-resolution imaging, although only the STORM images defined precisely their architecture (Fig. 4A). The pale core appeared unstained, while the peripheral dense halo was made of reticulated p.asyn. Likewise, STORM imaging of CLBs revealed dense honeycomb structures that could not be observed by conventional fluorescence microscopy (Fig. 4B,C). As for p.Tau aggregates, the unstained cores and spots observed in LBs might correspond to protein partners or trapped organelles. Indeed, Lewy bodies are known to be multiprotein complexes composed of more than 100 proteins, including p.Tau [10]. We thus performed two-color STORM imaging of LBs using antibodies against $p$.Tau and p.a-syn, to precisely define the internal architecture of the lesion and specifically distinguish one protein from the other (Fig. 5A). STORM imaging accurately measured the width of aggregated p.a-syn branches and the area of the unstained cores observed in CLBs, averaging $281.2 \pm 95.8 \mathrm{~nm}$ and $0.133 \pm 0.130 \mu \mathrm{m}^{2}$ respectively (mean $\pm S D$ ) (Fig. 5B). Finally, two-color STORM imaging of LNs revealed the internal organization of the neurites with a core of aggregated p.a-syn bounded to neurofilaments (Fig. 5C, Fig. S7). These very first STORM images of p.a-syn aggregates hold great promise for characterizing the composition and spatial organization of Lewy pathology in human brain.

\section{STORM imaging of TDP-43 neuronal inclusions}

Transactive response DNA-binding protein 43 (TDP-43), encoded by TARDBP gene, is a DNA/RNA binding protein predominantly located in the nucleus of cells under physiological condition, while the accumulation of misfolded TDP-43 in the cytosol and axons of degenerating neurons is a pathological hallmark of amyotrophic lateral sclerosis (ALS) and fronto-temporal lobar degeneration (FTLD) $[11,12]$. TDP-43 aggregates are called neuronal cytoplasmic inclusions $(\mathrm{NCl})$ when located in the cytosol, and dystrophic neurites (DN) when located in axons. The physiopathological mechanism leading to neuronal degeneration in both ALS and FTLD associates the cytoplasmic toxicity of aggregated TDP-43 and the nuclear loss of function of the protein. In frontal cortex sections from a control subject, STORM imaging revealed the presence of dense nuclear TDP-43 clusters while other nuclear zones were unstained, whereas the TDP-43 signal appeared diffuse and heterogeneous in conventional wide field fluorescence microscopy (Fig. 6A, Fig. S8). NCl and DN imaging with STORM in frontal and temporal cortex of patients affected with FTLD revealed compact and granular cytoplasmic structures, slightly denser and less reticulated than p.Tau and p.a-syn aggregates (Fig. 6B,C, Fig. S8). Interestingly, empty vacuoles were also defined in the core of $\mathrm{NCl}$ and $\mathrm{DN}$, suggesting again the presence of additional unstained components within these aggregates. Thus, STORM imaging of TDP-43 allowed to resolve the physiological 
distribution of the protein in the nuclear compartment, and its spatial organization within pathological cytoplasmic aggregates.

\section{Discussion}

In this work, we combined super resolution microscopy and neuropathological techniques to analyze human brain sections. This strategy did characterize with a nanometer-scale precision the architecture of physiological structures such as axons and synapses, and imaged with unprecedented details $A \beta, T a u, a-$ synuclein and TDP-43 pathological aggregates in samples from patients affected with neurodegenerative disorders.

To date, the main approach to image nanoscopic structures in tissues relies on transmission electron microscopy, a time-consuming technique which requires ultrathin tissue sections $(50-70 \mathrm{~nm})$ with stringent sample preparation, and limits immune-targeting diversity and 3D acquisition. Conversely, STORM offers the advantages of optical fluorescence microscopy with respect to sample preparation, vast observation fields, multiple molecular labelling and 3D acquisition, with image acquisition and reconstruction taking only a few minutes. The sample preparation workflow developed in this work for human brain tissue observation is timesaving and easily reproducible, as it is inspired by protocols routinely used for conventional fluorescence microscopy. It can be used on samples stored in a brain bank, with reagents and antibodies commonly used in clinical neuropathology departments and neuroscience research laboratories.

Nevertheless, performing STORM on human brain samples requires fine adjustments to generate high resolution images. Two major issues when imaging tissue sections are tissue autofluorescence and sample-induced aberrations that can distort and blur single-molecule emission patterns. Here, to limit optical artifacts and reduce the background generated by fluorophores located outside the focal plane, we used $5 \mu \mathrm{m}$ thin tissue sections and a TIRF illumination [13], where the incident laser beam is reflected at the coverslip and the sample is excited by the resulting evanescent wave. Imaging thicker brain sections could be considered but will require complementary techniques such as adaptive optics-based correction, self-interferences (SELFI) method or tissue clearing, to avoid excessive background and aberrations [14-16]. Sample preparation and immune-staining are also critical, the used fluorophores requiring high photoswitching kinetics and sufficient brightness to be detected properly. In this work, we used AF 647 and AF 532, as they exhibit appropriate photoswitching characteristics in antioxidant reagents. They also have long excitation and emission wavelengths, thus limiting background signal from tissue autofluorescence. Primary antibodies routinely used for immunostaining in clinical neuropathology departments have been used here for STORM, but the protocol can be adapted by using fluorescent probes or primary antibodies directly conjugated with fluorophores to limit the distance between the target antigens and the fluorophores and increase distance measurements accuracy.

To date, STORM has mainly been used to image nanoscale structures in cultured cells. However, the technique has recently been extended to rodent brain sections histological analysis. As in our study, two- 
color STORM allowed to visualize the molecular architecture of synapses, accurately identifying pre- and post-synaptic protein clusters and defining their size, morphology and orientation [5, 17-19]. STORM was also used to image pathological $A \beta$ aggregates in mouse models of Alzheimer's disease, leading to results comparable to ours in human, both for plaque morphologies and $A \beta$ fibril widths, ranging from 50 to $300 \mathrm{~nm}[15,20]$. Finally, STORM imaging of a-synuclein aggregates in a mouse model of Parkinson disease allowed to visualize a-synuclein aggregation in dopaminergic neurons [21], although the inclusions remained scattered and unstructured and no Lewy body was observed. These last results underline the relevance of human brain analyzes for studying the architecture and composition of pathological inclusions that cannot be induced in models.

To date, a single study reported STORM imaging on human brain tissue to visualize neurofilaments, myelin and astroglial processes within subcortical white matter [22]. This study evidenced axonal diameters in the same range as our measurements and TEM values, highlighting the reliability and reproducibility of the technique. Nevertheless, the authors faced limitations in sample preparation, hindering multiple immunostaining and limiting STORM acquisition to the white matter. Conversely, the protocol developed in this study allowed us to observe physiological and pathological structures in multiple areas of the human brain with a nanometer-scale precision.

The ultrastructural analysis of neuronal lesions is crucial to understand the pathogenesis and progression of neurodegenerative diseases. In this context and with the aim of improving neuropathological analyzes, the development of novel imaging approaches is a current challenge in neuroscience. For instance, the use of 3D multicolor STED microscopy correlative light and electron microscopy (CLEM) to analyze postmortem human brain tissue recently highlighted that Lewy bodies show an onion skin-like architecture with a Ser129-p aSyn external layer, and contain crowded membranous material from vesicles and fragmented organelles [23, 24]. Moreover, these techniques allowed to identify different pathological phenotypes of Lewy bodies within the CNS of the patients, which may reflect intracellular maturation stages of Lewy pathology [24]. Likewise, another new approach based on biochemical isolation of pathological TDP-43 recently provided evidence that aggregates extracted from patients with different subtypes of FTLD exhibited distinct morphological features and composition [25]. All these results indicate that the clinical and pathological heterogeneity observed in neurodegenerative disorders could originate from alternate ultrastructure and composition of neuronal lesions.

\section{Conclusions}

STORM imaging of fixed human tissue samples opens further gates to a more comprehensive understanding of the human brain organization, and revelations about the underlying mechanisms responsible for common neurological diseases. The convenience of this technique should open a straightforward extension of STORM applications for super-resolution imaging of human brain samples, with promising new avenues to current challenges in neuroscience. 


\section{Methods}

\section{Tissue samples}

All postmortem brain samples used for STORM imaging were obtained from the Neurodegenerative Diseases Brain Bank of Angers University Hospital. Six patients with neurodegenerative disorders and 3 age-matched control subjects were selected among the archived histological sections. Samples from prefrontal cortex, parietal cortex, temporal cortex, peri-ventricular white matter, and substantia nigra were considered. Clinical characteristics of patients and controls are reported in Table S3.

\section{Immunostaining}

Cryopreserved brain samples were used for STORM imaging (samples bathed 30 seconds into isopentane immersed in liquid nitrogen during brain autopsy and stored dry at $-80^{\circ} \mathrm{C}$ ). Tissue samples were cryo-sectioned at $-20^{\circ} \mathrm{C}$ into $5 \mu \mathrm{m}$ thick sections using a Leica CM3050 S cryostat (Leica Biosystems, Wetzlar, Germany) (Fig. S1a). Each frozen section was retrieved onto $22 \times 22 \mathrm{~mm} \mathrm{~N}{ }^{\circ} 1$ cover glass (Paul Marienfeld, Lauda-Königshofen, Germany), and air-dried for 45 minutes. After three washes in phosphate buffered saline (PBS) (PAN-Biotech, Aidenbach, Germany) the sections were blocked and permeabilized in $0.1 \%$ triton X-100 and 5\% bovine serum albumin (BSA) (Sigma-Aldrich, Saint-Louis, MO, USA) in PBS for $1 \mathrm{~h}$ at room temperature (RT). The sections were then washed and incubated over night at $4{ }^{\circ} \mathrm{C}$ with primary antibodies diluted in blocking solution. The next day, the sections were washed and incubated with appropriate AF-conjugated secondary antibodies (Thermo Fisher Scientific, Waltham, MA, USA) diluted in blocking solution for 2 hours at RT. TrueBlack ${ }^{\circledR}$ (Biotium, Fremont, CA, USA) was used to quench lipofuscin autofluorescence following manufacturer's instructions. References and dilutions of antibodies and reagents are reported in Table S4.

\section{STORM Acquisition}

For super resolution imaging, the cavity of a clean single depression slide (Paul Marienfeld, LaudaKönigshofen, Germany) was filled with $50 \mu \mathrm{L}$ of switching buffer (Abbelight, Paris, France), and covered by a coverslip, the sample side facing downward (Fig. S1b). Excess buffer was carefully wiped away, and the coverslip was sealed with a two-component silicone-glue (Twinsil ${ }^{\circledR}$, Picodent, Wipperfürth, Germany) (Fig. S1c). After a 10 minutes drying time, the device was placed on the stage of an inverted motorized microscope NIKON ECLIPSE Ti-E (Nikon Instruments Europe, Amsterdam, The Netherlands) equipped with a CFI SR APO TIRF 100X ON1.49 objective, a Perfect Focus System, and a total internal reflection fluorescence ILas2 module (Roper Scientific, Martinsried, Germany) (SI Appendix Fig. S2). Acquisition of images was proceeded using Metamorph 7.7 software (Molecular Devices, CA, USA). Image sequences were acquired with a single-photon sensitive camera Evolve 128TM EMCCD 512 ×512 imaging array, 16 $\times 16 \mu \mathrm{m}$ pixels (Photometrics, Tucson, AZ, USA). Acquisitions were performed at fixed $25^{\circ} \mathrm{C}$ in a dark heating chamber (Okolab NA, Pozzuoli, Italy). Phase contrast was first used for orientation and focus adjustment. The TIRF angle was then adjusted for each channel to reduce the background excitation. The region of interest was defined using the $647 \mathrm{~nm}$ and $532 \mathrm{~nm}$ laser line, and the $405 \mathrm{~nm}$ laser line was 
used to assess the autofluorescence signal. Prior to STORM imaging, a multichannel conventional fluorescence microscopy image was acquired for subsequent comparison with STORM image. The excitation power of either $647 \mathrm{~nm}$ or $532 \mathrm{~nm}$ laser line was then strongly increased ( 50 to $100 \mathrm{~mW}$ before the objective lens) to induce fluorophore blinking and preform STORM imaging. The em-gain of the camera was set to high amplification to optimize the signal-to-noise ratio. Images were acquired with an integration time of $30 \mathrm{~ms}$ per frame. The total acquisition time points for each sequence was adapted to the observed structure and to the labelling density (5,000 to 20,000 frames) (Video S1).

\section{Image reconstruction}

The center positions of the fluorophores were determined by fitting of 2D Gaussian function with parameters of microscope's point spread function (PSF) using WaveTracer software (Roper Scientific, Martinsried, Germany). For high-density STORM data sets (Video S3), we used a super-resolution radial fluctuation analytical approach to prevent artefacts due to fluorophores overcrowding [28]. Imaris 8.0 software (Bitplane, Zurich, Switzerland) was used for image processing and visualization, and Metamorph 7.7 (Molecular Devices, CA, USA) was used for measurements. To estimate the diameter of filamentous structures, cross-sectional widths were measured at multiple locations. To measure the area of the rounded structures, the border was manually defined, and the area was automatically calculated. More than 300 STORM images have been acquired and analyzed in total.

\section{Immunohistochemistry}

Formalin-fixed tissue samples were embedded in paraffin and cut at $4 \mu \mathrm{m}$ using a Leica RM125 microtome (Leica Biosystems, Wetzlar, Germany). Sections were collected on slides and immunostained using a fully automated IHC stainer Leica-BOND III (Leica Biosystems, Wetzlar, Germany). Finally, samples were analyzed under a Zeiss Axioscop40 microscope equipped with an Axio-cam MRC5 camera, using AxioVisio 4.6 software (Carl Zeiss, Oberkochen, Germany).

\section{Electron microscopy}

Brain cortical samples from the middle frontal gyrus of the right hemisphere were collected between 1974 and 1986 [29]. Biopsies were immediately fixed in Karnovsky's solution, postfixed in osmium, and embedded in Araldite. Samples from a 38-year-old male subject who suffered a brain hemorrhage were used as control (laboratory identification \#927). Samples from a 58-year-old male patient affected with a familial form of AD (M146L mutation of the PSEN1 gene) were used to study neurofibrillary tangles and senile plaques (laboratory identification \#1507). Seventy-nm-thick sections were collected on copper grids and contrasted by incubation with $5 \%$ uranyl acetate followed by lead citrate for 5 min each. Sections were analyzed with a Hitachi HT 7700 (Elexience, Verrieres le Buisson, France) electron microscope. More than 80 TEM images have been analyzed in total for each sample.

\section{Statistical analysis}

Statistical analyses were conducted using PRISM software version 5.0 for Windows (GraphPad, La Jolla, CA). Comparisons of means were performed using Student's t-test for two groups analysis, and one-way 
analysis of variance (ANOVA) for multiple groups analysis, after verifying the Normal distribution and the homoscedasticity of data using Shapiro-Wilk's test. In the absence of Normal distribution, the nonparametric Mann-Whitney U-test was used. Differences were considered to be significant at $p<0.01$.

\section{Abbreviations}

AB: Amyloid- $\beta$, AD: Alzheimer's disease, AF: Alexa Fluor, ALS: amyotrophic lateral sclerosis, BSA: bovine serum albumin, CLB: cortical Lewy bodies, CLEM: correlative light and electron microscopy, DLB:

dementia with Lewy bodies, DN: dystrophic neurites, EMCCD: electron multiplying charge coupled device, FTLD: fronto-temporal lobar degeneration: LB: Lewy bodies, LN: Lewy neurites, MBP: myelin basic protein, $\mathrm{NCl}$ : neuronal cytoplasmic inclusion, NF: neurofilaments, NFT: neurofibrillary tangles, p.a-syn:

phosphorylated a-synuclein, p.Tau: hyperphosphorylated tau protein, PBS: phosphate buffered saline, PD: Parkinson's disease, PSF: point spread function, RT: room temperature, SELFI: self-interferences, STORM: STochastic Optical Reconstruction Microscopy, TDP-43: Transactive response DNA-binding protein 43, TEM: transmission electron microscopy, TIRF: total internal reflection fluorescence, TLB: typical Lewy bodies.

\section{Declarations}

\section{Ethical approval and consent to participate}

Brain tissue samples were stored in the biological resource centre " Neurodegenerative disorders » (national identifier BB-0033-00038) after being authorized by the regional ethics committee West II (declaration number DC-2011-146). The study protocol has been declared to the French commission for information technology and civil liberties (declaration number ar19-0012v0). An anonymous database and a list of correspondence have been generated and stored on a hard disk of the University Hospital of Angers with restricted access.

\section{Consent for publication}

Not applicable.

\section{Availability of supporting data}

The data sets generated and analyzed during the current study are available from the corresponding author on reasonable request.

\section{Competing Interests}

The authors declare no competing interest.

\section{Funding}


This work was supported by the University Hospital of Angers (Grant N²019-264 900_036), the French National Institute for Health and Medical Research (INSERM Research Fellow 2017-2019), and the European Regional Development Fund (ERDF).

\section{Authors' Contributions}

P.C. initiated and led all aspects of the project, conducted the experiments, analyzed data and wrote the manuscript. A.C. and F.L. supervised the project. A.C. optimized the imaging protocol. F.L., L.R., C.D. and J.P.J. helped in developing the tissue preparation method. S.M. conducted TEM acquisitions. P.C., F.L., S.M., L.R., M.D., C.V., G.L., C.D., J.-P.J., J.C. and A.C. proofread the manuscript. All authors read and approved the final manuscript

\section{Acknowledgements}

The authors are grateful to the donors and their families. We also thank Dr Khalid Hamid El Hachimi for providing biopsies for electron microscopy, and C. Dumez, I. Viau and L. Denechaud for technical assistance.

\section{References}

1. Schermelleh L, Ferrand A, Huser T, Eggeling C, Sauer M, BiehImaier O, et al. Super-resolution microscopy demystified. Nat Cell Biol. 2019;21(1):72-84.

2. Rust MJ, Bates M, Zhuang X. Sub-diffraction-limit imaging by stochastic optical reconstruction microscopy (STORM). Nat Methods. 2006;3(10):793-795.

3. Sigal YM, Zhou R, Zhuang X. Visualizing and discovering cellular structures with superresolution microscopy. Science. 2018,361(6405):880-887.

4. Xu K, Zhong G, Zhuang X. Actin, spectrin, and associated proteins form a periodic cytoskeletal structure in axons. Science. 2013;339(6118):452-456.

5. Dani A, Huang B, Bergan J, Dulac C, Zhuang X. Superresolution imaging of chemical synapses in the brain. Neuron. 2010;68(5):843-856.

6. Huang $B$, Wang $W$, Bates $M$, Zhuang $X$. Three-dimensional super-resolution imaging by stochastic optical reconstruction microscopy. Science. 2008;319(5864):810-813.

7. Montine TJ, Phelps CH, Beach TG, Bigio EH, Cairns NJ, Dickson DW, et al. National Institute on AgingAlzheimer's Association guidelines for the neuropathologic assessment of Alzheimer's disease: a practical approach. Acta Neuropathol. 2012;123(1):1-11.

8. Schnell SA, Staines WA, Wessendorf MW. Reduction of lipofuscin-like autofluorescence in fluorescently labeled tissue. J Histochem Cytochem. 1999;47(6):719-730.

9. . Braak H, Del Tredici K. Invited Article: Nervous system pathology in sporadic Parkinson disease. Neurology. 2008;70(20):1916-1925. 
10. Arima K, Hirai S, Sunohara N, Aoto K, Izumiyama Y, Uéda K, et al. Cellular co-localization of phosphorylated tau- and NACP/alpha-synuclein-epitopes in lewy bodies in sporadic Parkinson's disease and in dementia with Lewy bodies. Brain Res. 1999;843(1-2):53-61.

11. Arai T, Hasegawa M, Akiyama H, Ikeda $K$, Nonaka T, Mori H, et al. TDP-43 is a component of ubiquitin-positive tau-negative inclusions in frontotemporal lobar degeneration and amyotrophic lateral sclerosis. Biochem Biophys Res Commun. 2006;351(3):602-611.

12. Neumann M, Sampathu DM, Kwong LK, Truax AC, Micsenyi MC, Chou TT, et al. Ubiquitinated TDP-43 in frontotemporal lobar degeneration and amyotrophic lateral sclerosis. Science. 2006;314(5796):130-133.

13. Boulanger J, Gueudry C, Münch D, Cinquin B, Paul-Gilloteaux P, Bardin S, et al. Fast high-resolution 3D total internal reflection fluorescence microscopy by incidence angle scanning and azimuthal averaging. Proc Natl Acad Sci U S A. 2014;111(48):17164-17169.

14. Bon $P$, Linarès-Loyez $J$, Feyeux $M$, Alessandri $K$, Lounis $B$, Nassoy $P$, et al. Self-interference $3 D$ superresolution microscopy for deep tissue investigations. Nat Methods. 2018;15(6):449-454.

15. Mlodzianoski MJ, Cheng-Hathaway PJ, Bemiller SM, McCray TJ, Liu S, Miller DA, et al. Active PSF shaping and adaptive optics enable volumetric localization microscopy through brain sections. Nat Methods. 2018;15(8):583-586.

16. Gustavsson AK, Petrov PN, Moerner WE. Light sheet approaches for improved precision in 3D localization-based super-resolution imaging in mammalian cells. Opt Express. 2018;26(10):1312213147.

17. Schoen M, Reichel JM, Demestre M, Putz S, Deshpande D, Proepper C, et al. Super-Resolution Microscopy Reveals Presynaptic Localization of the ALS/FTD Related Protein FUS in Hippocampal Neurons. Front Cell Neurosci. 2016;9:496.

18. Sigal YM, Speer CM, Babcock HP, Zhuang X. Mapping Synaptic Input Fields of Neurons with SuperResolution Imaging. Cell. 2015;163(2):493-505.

19. Dudok B, Barna L, Ledri M, Szabó SI, Szabadits E, Pintér B, et al. Cell-specific STORM super-resolution imaging reveals nanoscale organization of cannabinoid signaling. Nat Neurosci. 2015;18(1):75-86.

20. Yuan P, Condello C, Keene CD, Wang Y, Bird TD, Paul SM, et al. TREM2 Haplodeficiency in Mice and Humans Impairs the Microglia Barrier Function Leading to Decreased Amyloid Compaction and Severe Axonal Dystrophy. Neuron. 2016;90(4):724-739.

21. Wegrzynowicz M, Bar-On D, Calo' L, Anichtchik O, lovino M, Xia J, et al. Depopulation of dense asynuclein aggregates is associated with rescue of dopamine neuron dysfunction and death in a new Parkinson's disease model. Acta Neuropathol. 2019;138(4):575-595.

22. Hainsworth AH, Lee S, Foot P, Patel A, Poon WW, Knight AE. Super-resolution imaging of subcortical white matter using stochastic optical reconstruction microscopy (STORM) and super-resolution optical fluctuation imaging (SOFI). Neuropathol Appl Neurobiol. 2018;44(4):417-426. 
23. Shahmoradian SH, Lewis AJ, Genoud C, Hench J, Moors TE, Navarro PP, et al. Lewy pathology in Parkinson's disease consists of crowded organelles and lipid membranes. Nat Neurosci. 2019;22(7):1099-1109.

24. Moors TE, Maat CA, Niedieker D, Mona D, Petersen D, Timmermans-Huisman E,et al. Subcellular orchestration of alpha-synuclein variants in Parkinson's disease brains revealed by 3D multicolor STED microscopy. bioRxiv. 2019;470476. https://www.biorxiv.org/content/10.1101/470476v3.

25. Laferrière F, Maniecka Z, Pérez-Berlanga M, Hruska-Plochan M, Gilhespy L, Hock EM, et al. TDP-43 extracted from frontotemporal lobar degeneration subject brains displays distinct aggregate assemblies and neurotoxic effects reflecting disease progression rates. Nat Neurosci. 2019;22(1):6577.

26. Braak H, Del Tredici K, Rüb U, de Vos RA, Jansen Steur EN, Braak E. Staging of brain pathology related to sporadic Parkinson's disease. Neurobiol Aging. 2003;24(2):197-211.

27. Mackenzie IR, Neumann M, Baborie A, Sampathu DM, Du Plessis D, Jaros E, et al. A harmonized classification system for FTLD-TDP pathology. Acta Neuropathol. 2011;122(1):111-113.

28. Gustafsson N, Culley S, Ashdown G, Owen DM, Pereira PM, Henriques R. Fast live-cell conventional fluorophore nanoscopy with ImageJ through super-resolutionradial fluctuations. Nat Commun. 2016;7:12471.

29. Androuin A, Potier B, Nägerl UV, Cattaert D, Danglot L, Thierry $M$, et al. Evidence for altered dendritic spine compartmentalization in Alzheimer's disease and functional effects in a mouse model. Acta Neuropathol. 2018;135(6):839-854.

\section{Supplementary Legends}

\section{Supplementary Figure Legends}

Fig. S1. Sample preparation work-flow for STORM acquisition. a, Cryopreserved brain samples (black arrowhead) were cryo-sectioned at $-20{ }^{\circ} \mathrm{C}$ into $5 \mu \mathrm{m}$ thick sections and retrieved onto $22 \times 22 \mathrm{~mm} \mathrm{~N} 1$ cover glasses. b, Once the sample immunostained, the cavity of a clean single depression slide was filled with $50 \mu$ of switching buffer (black arrowhead) and covered by the coverslip. c, Excess buffer was then carefully wiped away, and the coverslip was sealed on the slide with a two-component silicone-glue.

Fig. S2. Detailed microscope setup used for STORM imaging. a, Inverted motorized microscope NIKON ECLIPSE Ti-E. b, Central computer equipped with Metamorph 7.7 software. c, Dark heating chamber. $d$, Evolve 128TM EMCCD camera. e, Andor NEO sCOMS camera. f, Anti-vibration table. g, LED bench. h, Piezoelectric driver system. i, Total Internal Reflection Fluorescence (TIRF) module. j, Laser bench.

Fig. S3. Conventional wide field fluorescence and STORM imaging of the same axonal process. (A1) Wide field fluorescence image of a longitudinally sectioned axon immunostained for neurofilaments (NF) in prefrontal cortex. (A2) STORM image of the same area. (B) Representation of the cross-sectional profiles 
(dotted lines in a) through axonal process. Error bars indicate mean with standard error, $\mathrm{n}=8$ measures per image.

Fig. S4. Individual color channel sequences of the composites images in Fig. 2B and C.

Fig. S5. Individual color channel sequences of the composites images in Fig. 3B.

Fig. S6. (A) Conventional wide field fluorescence and STORM imaging of the same amyloid plaque. (B) $A \beta$ fibrils width in senile plaques measured in STORM images.

Fig. S7. Individual color channel sequences of the composites images in Fig. 5B.

Fig. S8. Delimitation of the nuclear compartment in neurons (Fig. 6A and B) using Lamin A/C signal.

\section{Supplementary Video Legends}

Supplementary Video 1. STORM imaging of a cortical axon in a human brain section immunostained for neurofilaments.

Supplementary Video 2. 3DSTORM imaging of a cortical axon in a human brain section immunostained for neurofilaments.

Supplementary Video 3. Representative STORM acquisition of a structure with high-density emitting fluorophores (cortical Lewy body immunostained for a-synuclein).

\section{Figures}


A

Microscope Slide

TIRF Objective

Laser Module

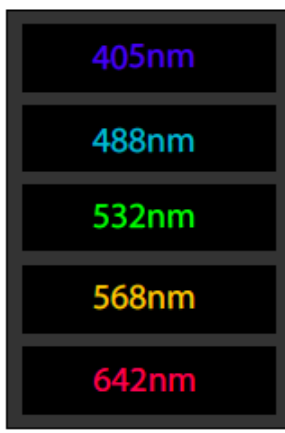

B Wide Field

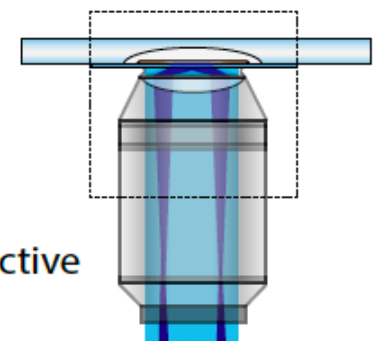

Dichroic

Mirror

Emission

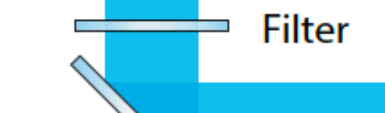

Mirror

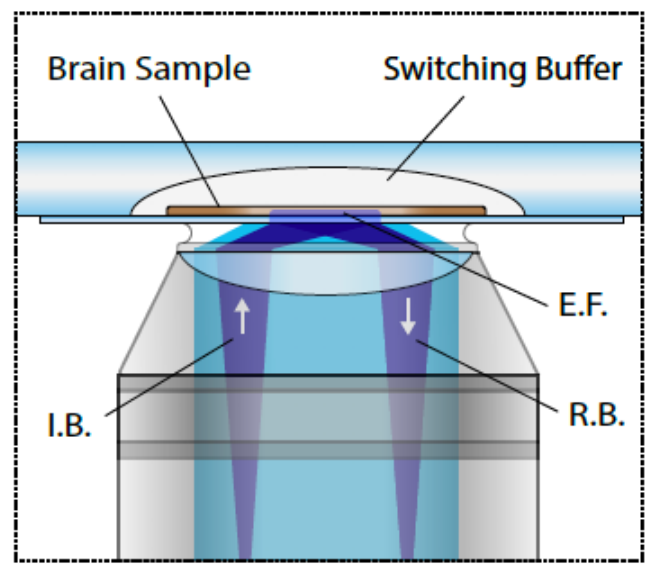

EMCCD

Camera

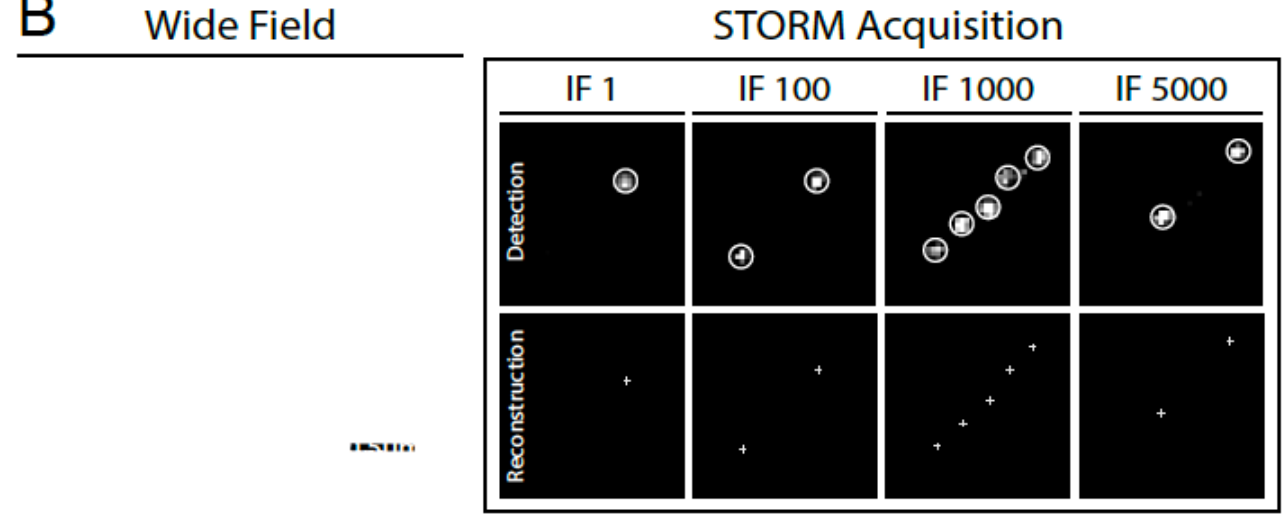

\section{Wide Field}
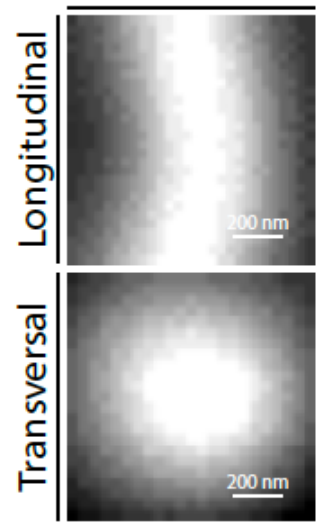
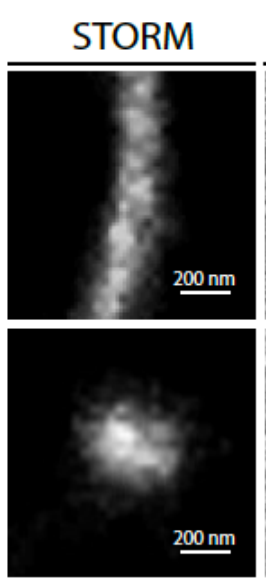

TEM

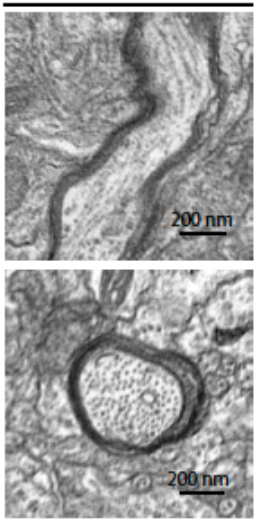

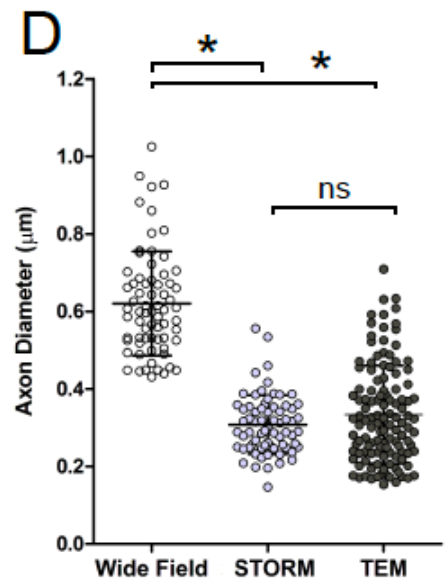

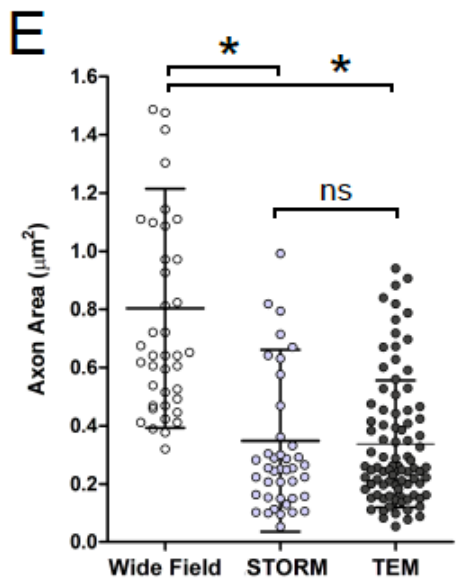

\section{Figure 1}

Super-resolution imaging of human brain samples with STORM. (A) Schematic of the optical setup used for STORM imaging. I.B. = incident beam, E.F = evanescent field, R.B. = reflected beam. (B) STORM acquisition of a cortical axon in a human brain section immuno-stained for neurofilaments (NF): a conventional wide field fluorescence microscopy image was first acquired (left), excitation power was then strongly increased to induce fluorophore blinking and thousands of frames were recorded (center - 
up). Each frame was computationally processed to detect activated fluorescent molecules and report their center position as single pixels on a corresponding reconstruction picture (center - down). The final STORM image was obtained by merging all the reconstruction pictures in a single overlap image (right). IF = imaging frame. (C) Representative images of longitudinally- and transversally-sectioned prefrontal cortex axons acquired with conventional wide field fluorescence microscopy, STORM, and transmission electron microscopy (TEM). (D and E) Axon diameters (longitudinal sections) and areas (transversal sections) measured in human brain using conventional fluorescence microscopy, STORM, and TEM. Error bars indicate means with standard deviations. ${ }^{*} p<0.001$. 

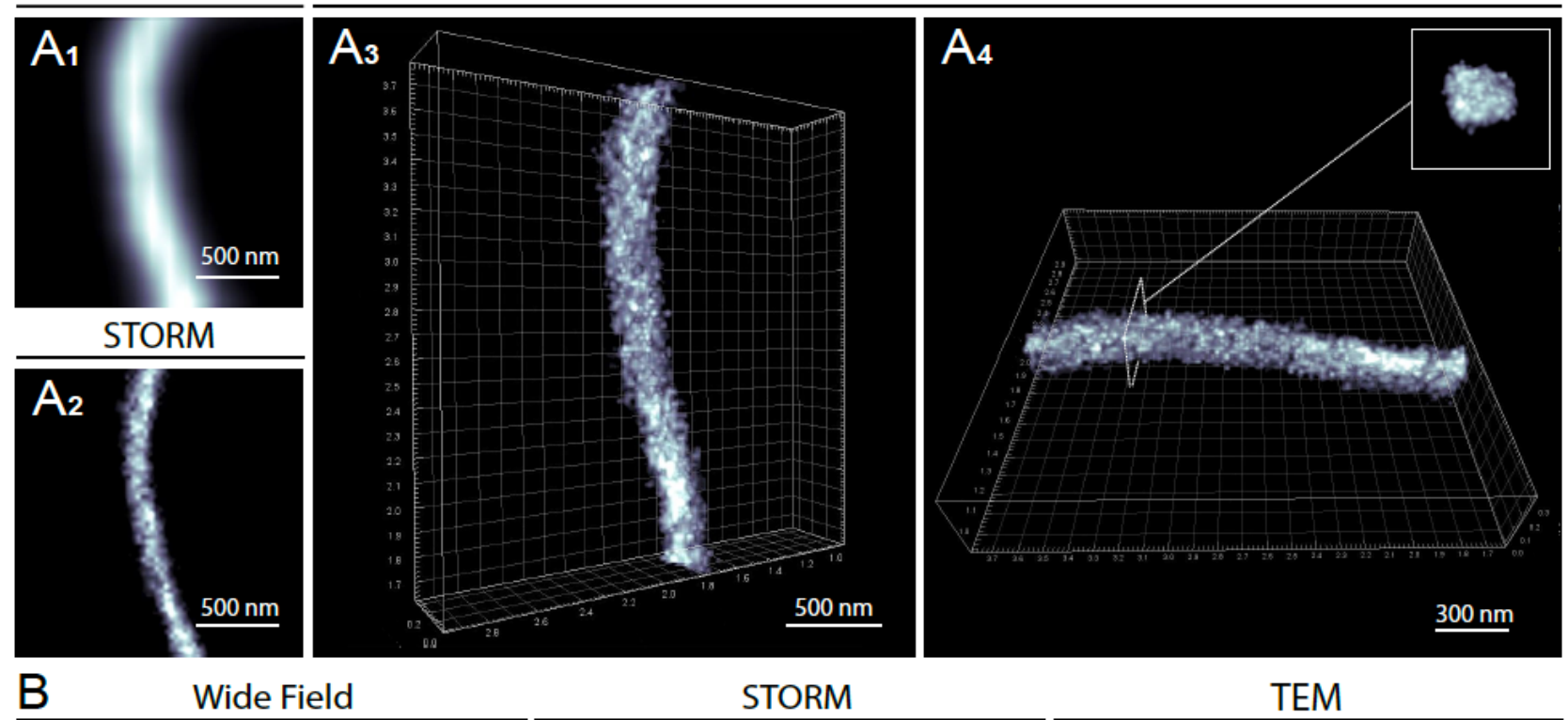

STORM
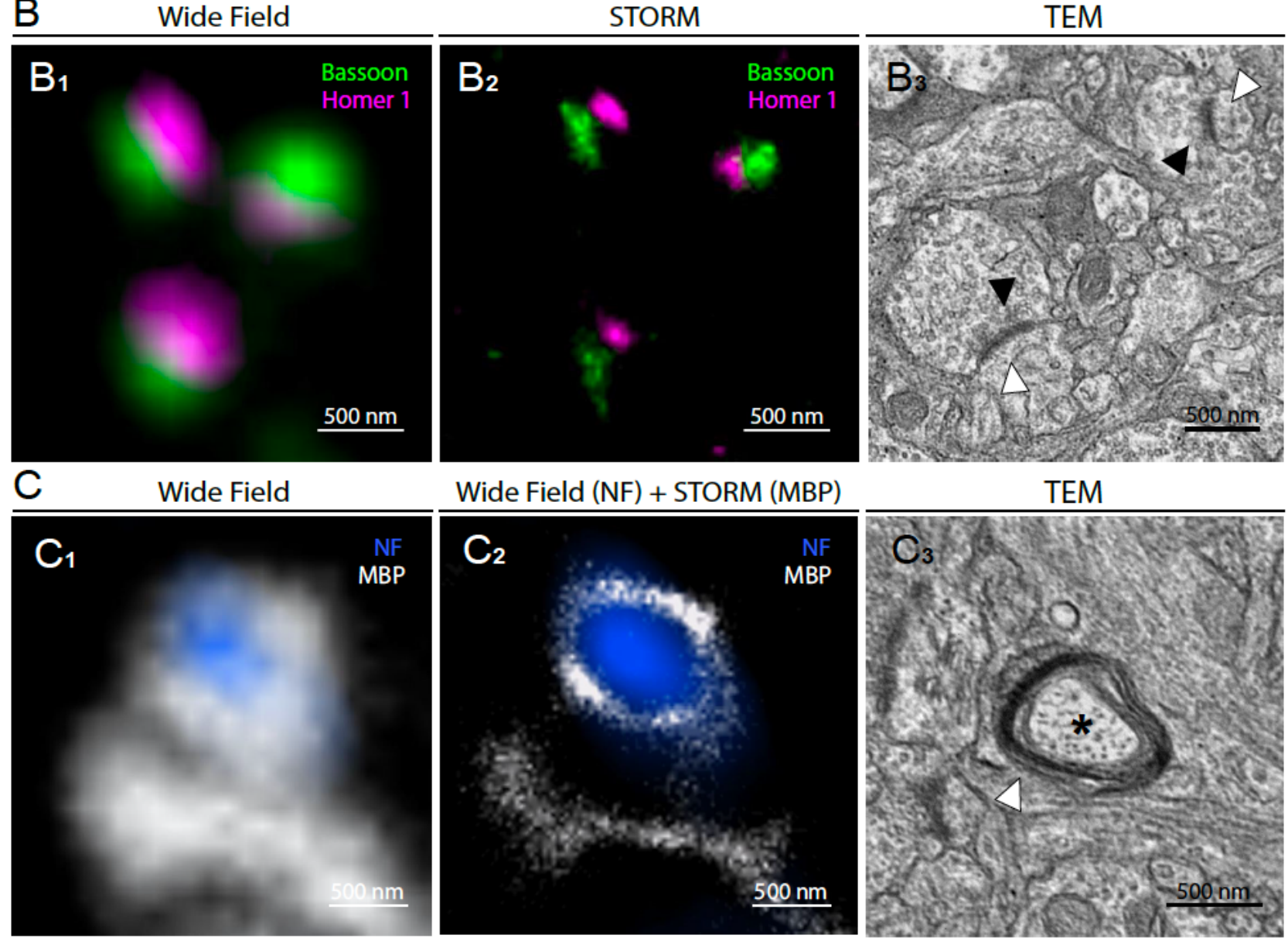

Wide Field (NF) + STORM (MBP)

TEM
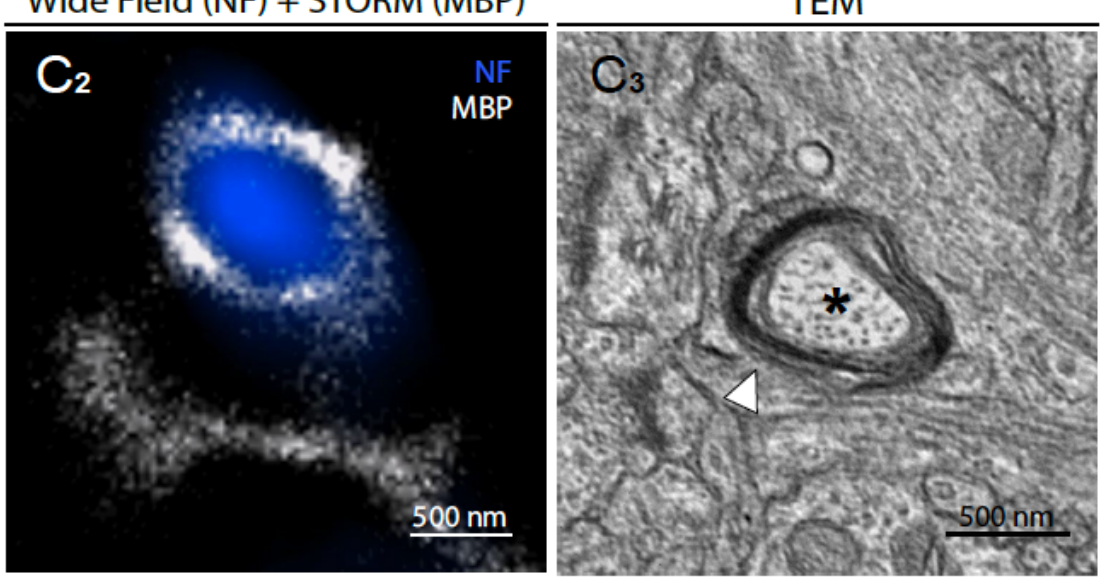

\section{Figure 2}

3D-STORM and two-color STORM images of physiological structures in human brain. (A1) Conventional fluorescence microscopy image of a longitudinally sectioned axon immunostained for neurofilament (NF) in prefrontal cortex. (A2) STORM image of the same area. (A3 and A4) 3D-STORM reconstruction of the acquired axon. (B1) Conventional fluorescence microscopy image of pre- and post-synaptic proteins Bassoon and Homer 1 in prefrontal cortex. (B2) Two-color STORM image of the same area resolving 
distinct synaptic clusters. (B3) Comparative TEM image of 2 synapses with pre- (black arrowheads) and post- (white arrowheads) synaptic compartments. (C1) Conventional fluorescence microscopy image of axonal NF and oligodendrocyte myelin basic protein (MBP) in peri-ventricular white matter. (C2) Image of the same area combining conventional fluorescence microscopy (NF) and STORM (MBP). (C3) Comparative TEM image of a transversally sectioned axon (asterisk) surrounded by oligodendrocyte processes and myelin sheath (white arrowhead).

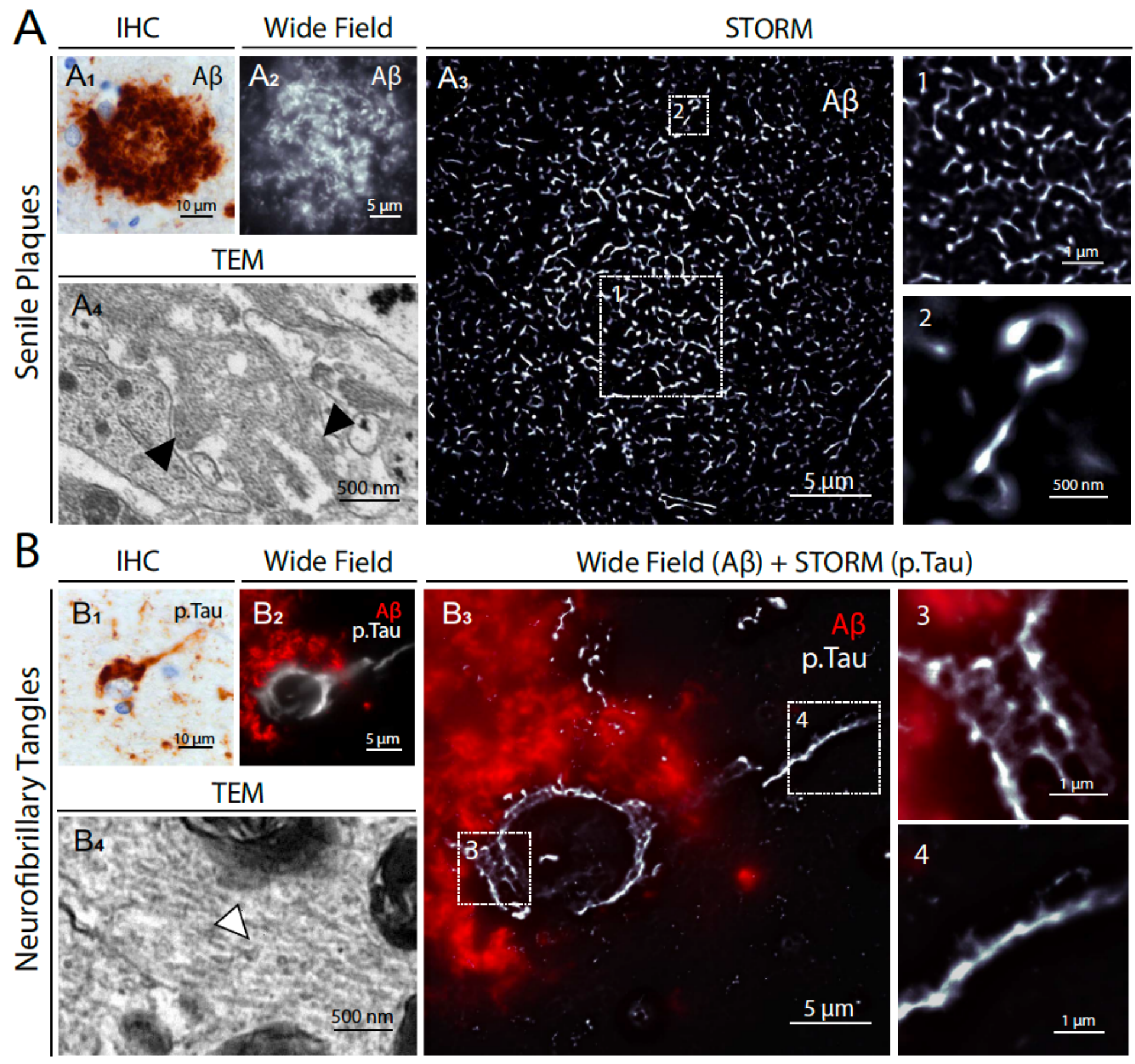

Figure 3

STORM images of senile plaques and neurofibrillary tangles in AD patient brain samples. (A1) Representative image of a senile plaque in the neocortex of an AD patient (immunohistochemical 
detection of $A B$ ). (A2) Conventional fluorescence microscopy image of a whole senile plaque in a neocortex section of the same patient immunostained for A 3 . (A3) STORM image of the same area. The insets ( 1 and 2 ) show close-up details of the distribution and size of aggregated $A \beta$ branches. (A4) Comparative TEM image of $A \beta$ fibrils (black arrowheads) in a senile plaque. (B1) Representative image of neurofibrillary tangles in the neocortex of an AD patient (immunohistochemical detection of p.Tau). (B2) Conventional fluorescence microscopy image of neurofibrillary tangles within the soma of a whole degenerating neuron surrounded by $A \beta$ deposition in a neocortex section of the same patient. (B3) Same neuron imaged by combining conventional fluorescence microscopy (A $\beta$ ) and STORM (p.Tau). The insets (3 and 4) show close-up details of the honeycombed structure of p.Tau aggregates in the soma and the filamentous organization in the axon. (B4) Comparative TEM image of Tau filaments (white arrowhead) in neurofibrillary tangles. 

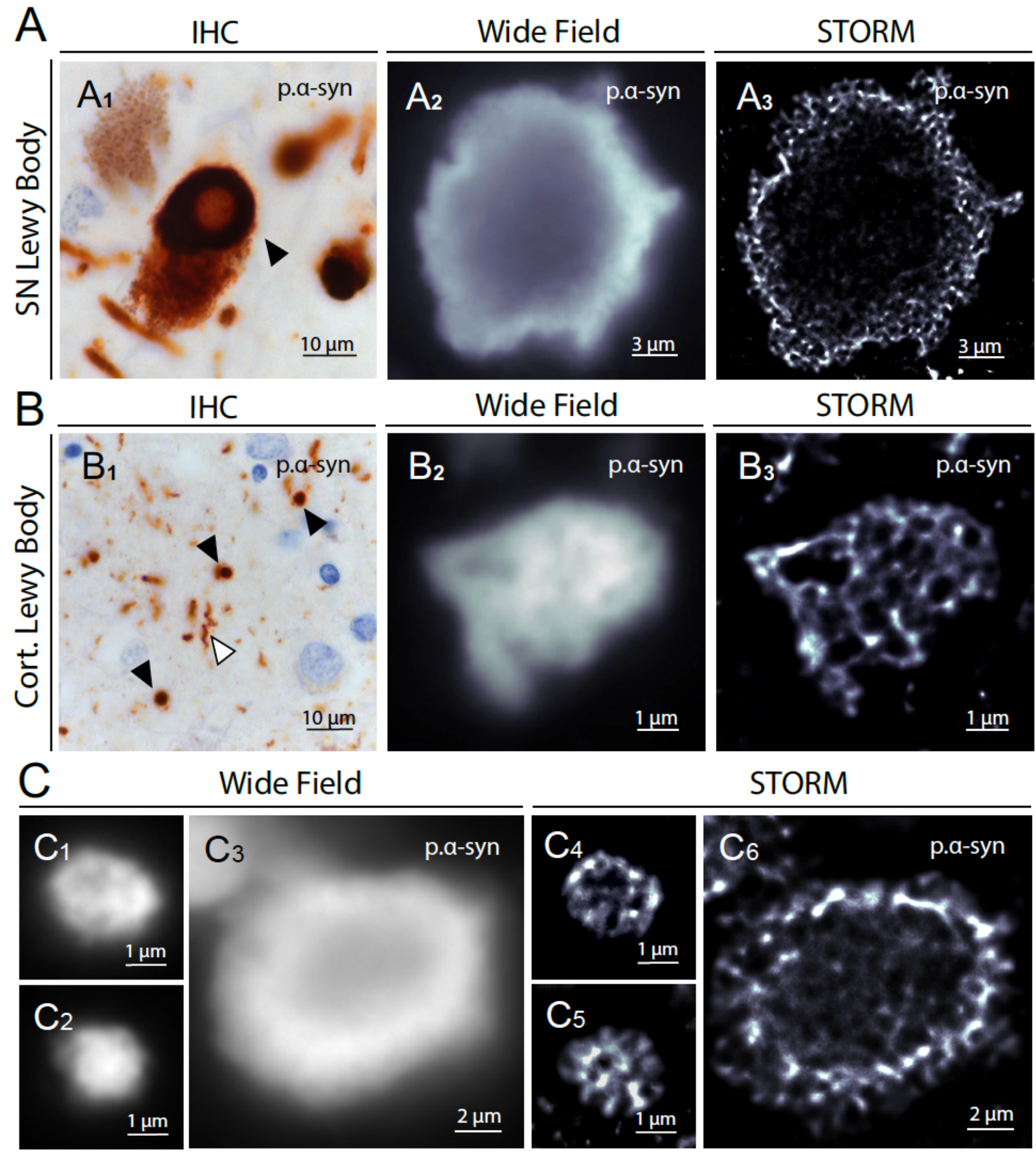

\section{STORM}

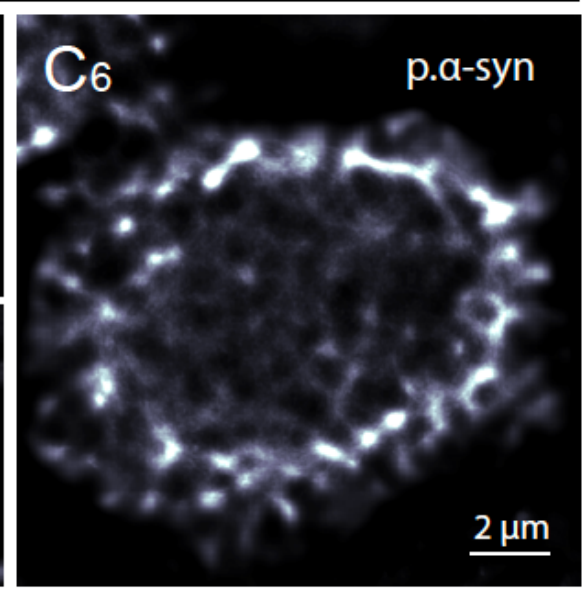

Figure 4

STORM images of Lewy bodies in PD and DLB patients brain samples. (A1) Representative image of a typical Lewy body (black arrowhead) in the substantia nigra of a PD patient (immunohistochemical detection of p.a-syn). (A2) Conventional fluorescence microscopy image of a Lewy body in a substantia nigra section of the same patient immunostained for p.a-syn. (A3) STORM image of the same LB resolving distinctly the asynuclein reticulated corona and the unstained core. (B1) Representative image 
of cortical Lewy bodies (black arrowheads) and Lewy neurite (white arrowhead) in the neocortex of an PD patient. (B2) Conventional fluorescence microscopy image of a cortical Lewy body in a neocortex section of the same patient immunostained for p.a-syn. (B3) STORM image of the same area revealing the dense honeycombed organization of the Lewy body. (C) Conventional fluorescence microscopy representative images of cortical (C1 and C2) and SN (C3) Lewy bodies immunostained for p.a-syn. STORM images of the same areas $(\mathrm{C} 4, \mathrm{C} 5$ and $\mathrm{C} 6)$ highlight the distinct internal organization of the protein aggregates.

A Wide Field

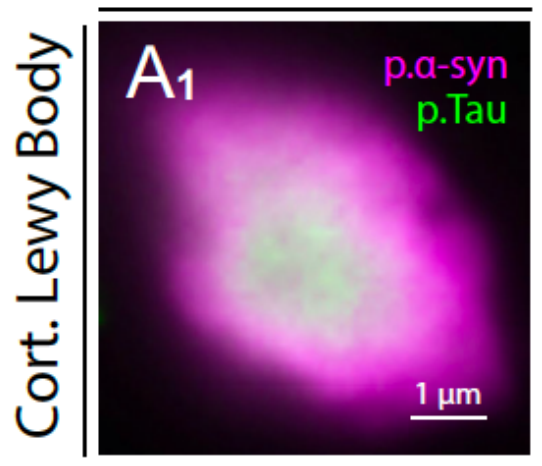

$\mathrm{B}$

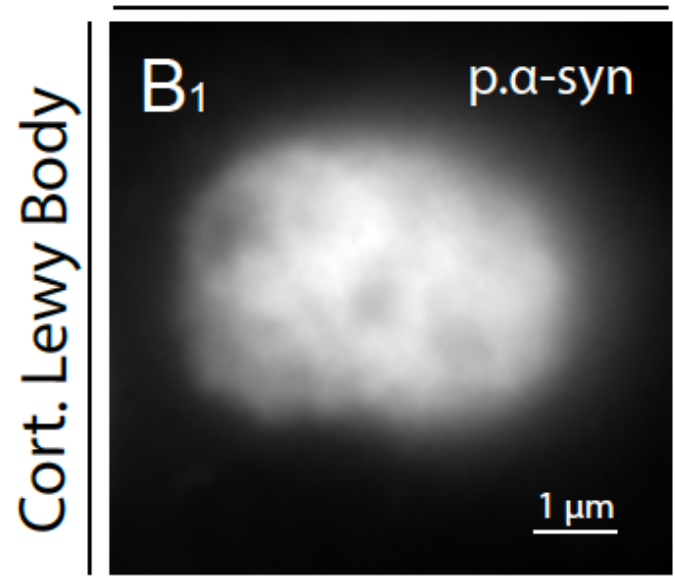

C

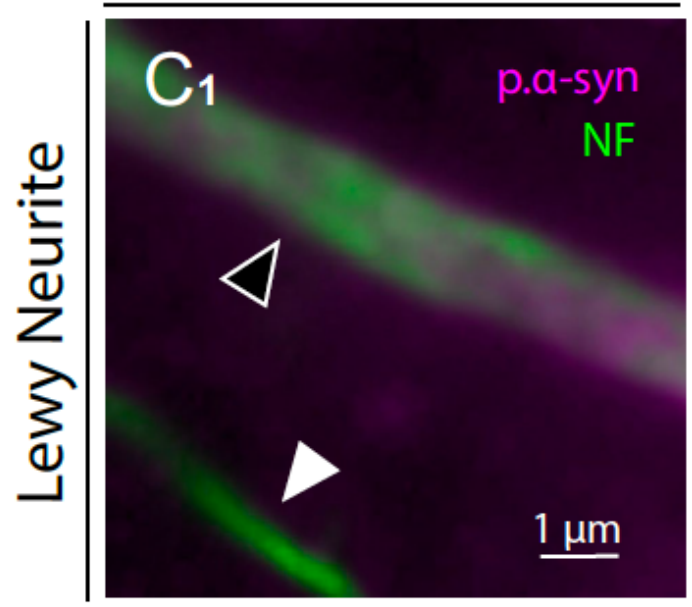

STORM

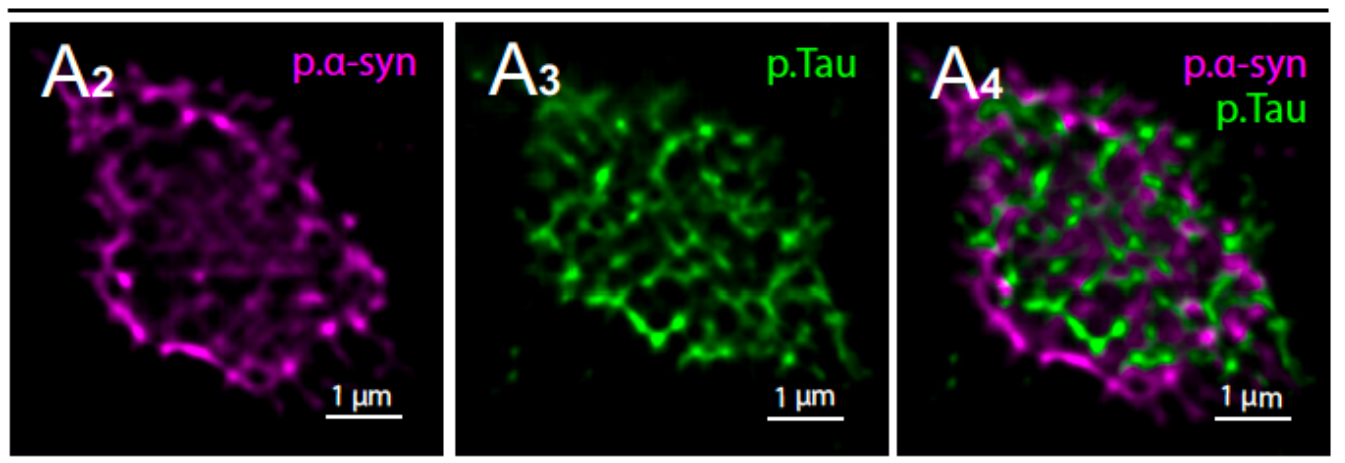

STORM
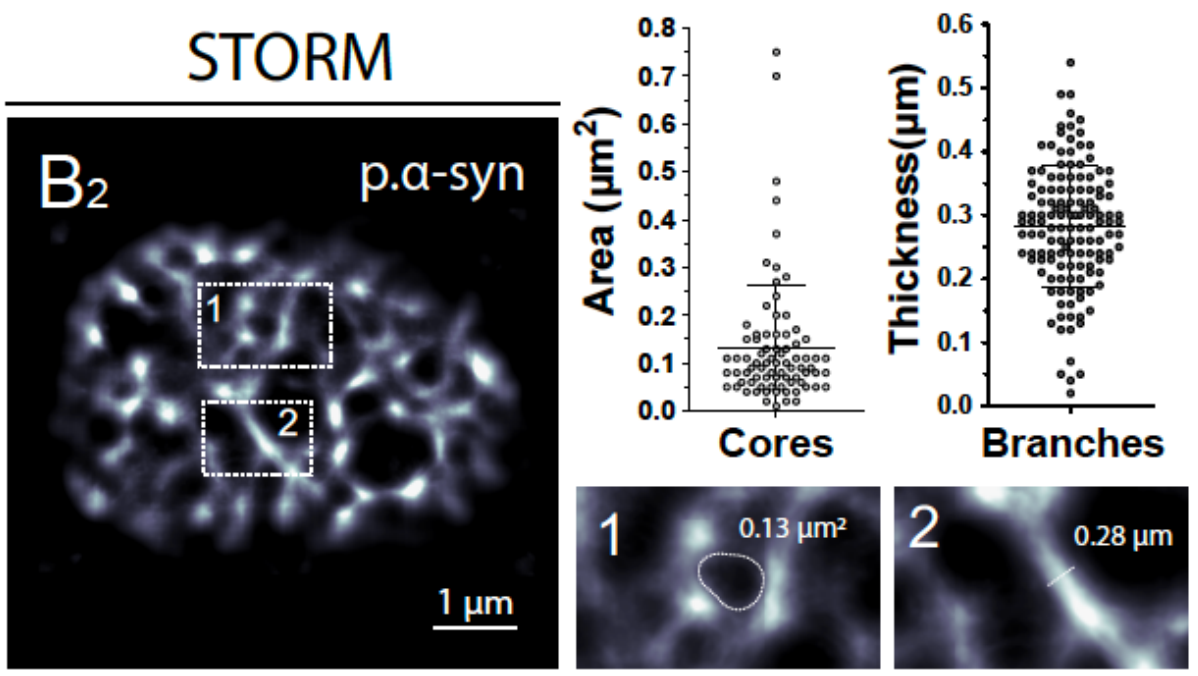

STORM

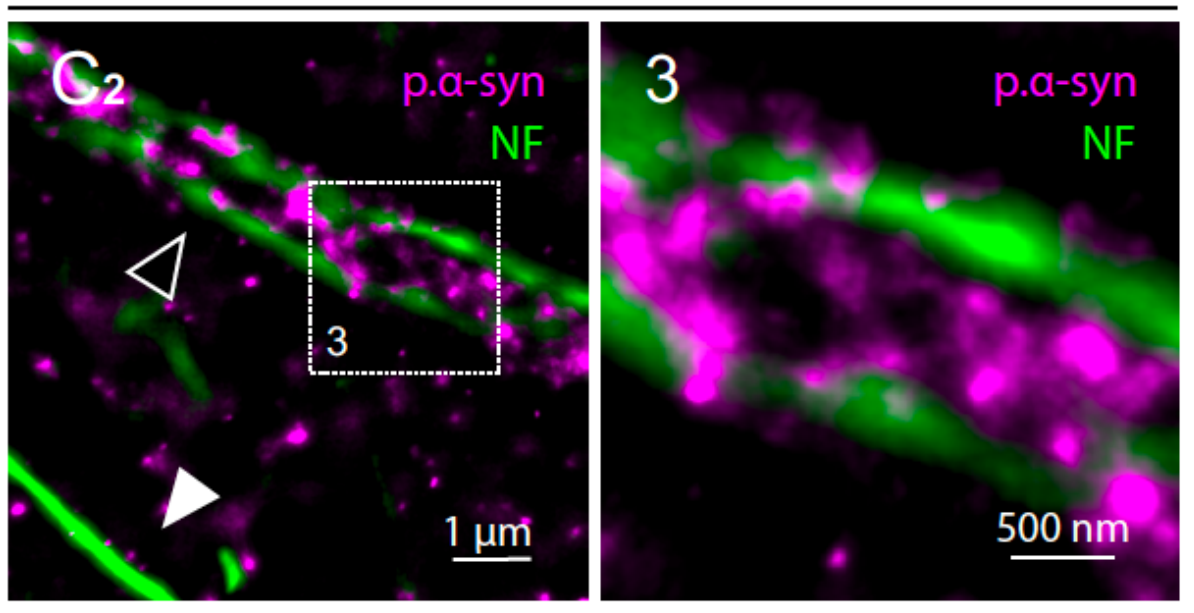

Figure 5 
Colocalization and ultrastructural analysis of Lewy bodies and Lewy Neurites. (A1) Conventional fluorescence microscopy image of a cortical Lewy body immunostained for p.a-syn and p.Tau. (A2-A4) Two-Color STORM image of the same area revealing the internal architecture of the lesion and allowing to distinguish one protein from the other. (B) Ultrastructural analysis of a cortical Lewy body (B1) using STORM (B2). Areas of the unstained cores and widths of p.a-syn branches were measured on superresolved images. Error bars indicate means with standard deviations. (C1) Conventional fluorescence microscopy image of a Lewy neurite (black arrowhead) immunostained for p.a-syn and neurofilaments (NF). (C2) Two-Color STORM image of the same area showing p.a-syn dense aggregates bounded by neurofilaments (enlarged in 3). Note the unaffected axon with normal caliber (white arrowhead). 


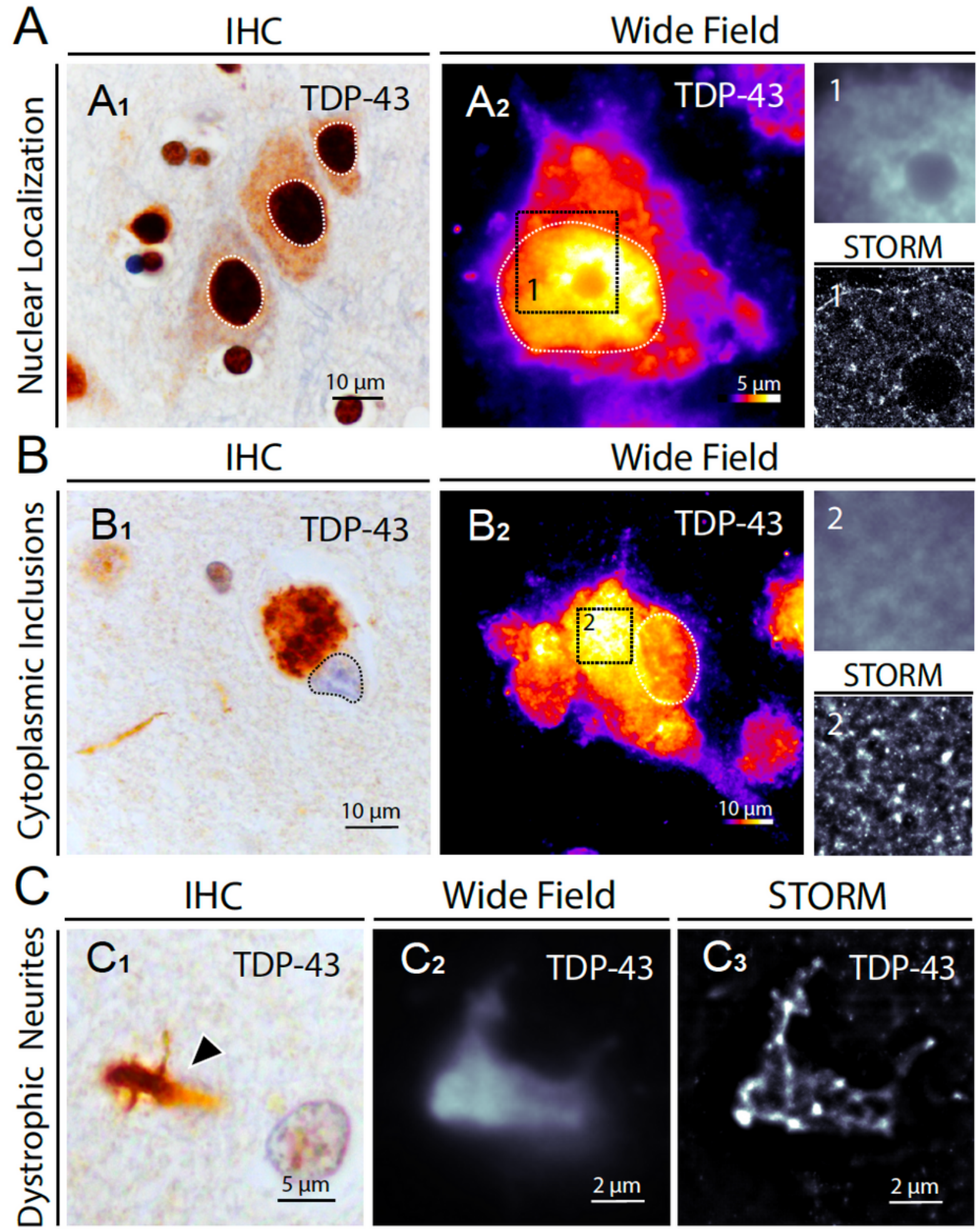

Figure 6

STORM images of TDP-43 aggregates in FTLD brain samples. (A1) Representative image of normal TDP43 localization in cortical neurons (immunohistochemical detection of TDP-43 in the prefrontal cortex of a control subject). (A2) Conventional fluorescence microscopy image of TDP-43 distribution in a nondegenerating neuron in the prefrontal cortex of the same subject (signal intensity scale ranging from 1 to $254 \mathrm{UI}$ ). Nuclear compartments are defined with dotted lines. The insets (1) show close-up details of TDP- 
43 distribution within the nucleus as assessed by conventional wide field fluorescence microscopy (up) and STORM (down). (B1) Representative image of TDP-43 cytoplasmic inclusions in a cortical neuron (immunohistochemical detection of TDP-43 in the prefrontal cortex of a FTLD patient). (B2) Conventional fluorescence microscopy image of TDP-43 distribution in a degenerating neuron in the prefrontal cortex of the same patient (signal intensity scale ranging from 1 to $254 \mathrm{UI}$ ). Nuclear compartments are defined with dotted lines. The insets (2) show close-up details of TDP-43 aggregate as assessed by conventional wide field fluorescence microscopy (up) and STORM (down). (C) Representative light microscopy, wide field fluorescence microscopy and STORM images of TDP-43 aggregates in dystrophic neurites (black arrowhead in C1) in the prefrontal cortex of a FTLD patient.

\section{Supplementary Files}

This is a list of supplementary files associated with this preprint. Click to download.

- Additionalfile1Figures1.pdf

- Additionalfile15VideoS3.mp4

- Additionalfile10FigureS6.pdf

- Additionalfile11FigureS7.pdf

- Additionalfile12FigureS8.pdf

- Additionalfile13Tables3.docx

- Additionalfile14Tables4.docx

- Additionalfile8Figures4.pdf

- Additionalfile9Figures5.pdf

- Additionalfile5Tables1.docx

- Additionalfile6Tables2.docx

- Additionalfile7VideoS2.mp4

- Additionalfile3VideoS1.mp4

- Additionalfile4Figures3.pdf

- Additionalfile2Figures2.pdf 